\title{
Asymptomatic superior mesenteric vein thrombosis as unusual complication of acute cytomegalovirus infection: a case report
}

\author{
Michele Bertoni, Filippo Risaliti, Alessandra Giani, Emanuele Calabrese, Massimo Edoardo Di Natale \\ $2^{\text {nd }}$ Unit of Internal Medicine, Santo Stefano New Hospital of Prato, Prato, Italy
}

\begin{abstract}
We describe a 39-year-old male who presented with a fever of unknown origin, the diagnostic work-up of which disclosed an acute cytomegalovirus (CMV) infection complicated by a partial superior mesenteric vein (SMV) thrombosis. Further investigations revealed the presence of factor V Leiden mutation. Oral anticoagulant treatment with warfarin led to a complete recanalization of SMV two months after. A literature review on the association between CMV infection and portal system (PS) thrombosis in immunocompetent patients was performed. We found that, in agreement with our case, in a minority of case reports patients did not complain of abdominal pain, but presented with a mononucleosis-like syndrome with malaise and prolonged fever and displayed a variable elevation of aminotransferase levels. Interestingly, most of them exhibited a limited extension of portal thrombosis. On the whole, these data suggest that PS thrombosis during acute CMV infection may be an underestimated complication.
\end{abstract}

\section{Introduction}

Acute cytomegalovirus (CMV) infection is usually asymptomatic in immunocompetent patients or may cause a benign viral syndrome in about $10 \%$ of them. ${ }^{1}$ Severe complications, such as venous thrombosis, have been rarely reported although they may be underestimated. ${ }^{2}$ In this regard, a recent prospective study has suggested that acute CMV infection may be

Correspondence: Michele Bertoni, $2^{\text {nd }}$ Division of Internal Medicine, Santo Stefano New Hospital of Prato, via Ugo Foscolo 5, 59100 Prato, Italy.

Tel.: +39.0574.804308 - Fax: +39.0574.802977.

E-mail: micbertoni@libero.it

Key words: Cytomegalovirus infection; superior mesenteric vein thrombosis; pro-thrombotic risk factors.

Contributions: MB, case management, manuscript writing, acquisition of images; FR, AG, search for references and manuscript review; MED, EC, case management and manuscript review.

Conflict of interest: the authors declare no conflict of interest.

Received for publication: 17 February 2015.

Revision received: 25 May 2015.

Accepted for publication: 18 August 2015.

This work is licensed under a Creative Commons Attribution NonCommercial 4.0 License (CC BY-NC 4.0).

CC Copyright M. Bertoni et al., 2016

Licensee PAGEPress, Italy

Italian Journal of Medicine 2016; 10:147-150

doi:10.4081/itjm.2015.596 associated with an increased short-term risk of venous thrombosis. ${ }^{3}$ In immunocompetent patients with CMV-associated venous thrombosis, the portal system (PS) seems to be less commonly involved than lower limb deep venous district and pulmonary circulation. ${ }^{4}$ In this paper, we describe an immunocompetent man who presented with a fever of unknown origin, the diagnostic work-up of which disclosed an acute CMV infection complicated by superior mesenteric vein (SMV) thrombosis. Then, we discuss this case in light of a review of the literature and we focus on the effects of CMV infection on coagulation pathways.

\section{Case Report}

A 39-year-old man with a negative history of thromboembolic disease was admitted to our division because of a 4-week persistent fever, weakness and a mild headache. The physical examination was normal with the exception of body temperature $\left(38.5^{\circ} \mathrm{C}\right)$ and a mild throat erythema. Laboratory data on admission revealed lymphocytosis [6200 cells/mL, normal range (n.r.) 6003400] with atypical lymphocytes, as well as an increase in erythrocyte sedimentation rate $(53 \mathrm{~mm} / \mathrm{hr}$, n.r. 1-20), $\mathrm{C}$ reactive protein $(3.91 \mathrm{mg} / \mathrm{dL}$, n.r. $0-0.50)$, aspartate aminotransferase (48 U/L, n.r. 0-40), alanine aminotransferase (54 U/L, n.r. 0-35), lactic dehydrogenase (429 U/L, n.r. 130-250), and gamma glutamyl transpeptidase (196 U/L, n.r. 7-49) levels. A subsequent series of laboratory tests was therefore performed and evidenced an increase in serum total immunoglobulin (Ig) M levels (546 mg/dL, n.r. 34-210) and CMV-IgM antibodies (3.31 ELFA Index, n.r. $<0.70$ ), as well as the positivity of the polymerase chain reaction CMV-DNA 
assay (256 copies $/ \mathrm{mL}$ ), while CMV-IgG antibodies were negative. Further serological tests were negative for Epstein-Barr virus, hepatitis A, B, C viruses, and HIV infection. Blood and urine cultures were negative. The study of lymphocytic immunophenotype revealed an increase in CD8+ T cells $(3866$ cells $/ \mathrm{mL}$, n.r. 10001850 ), and natural killer cells ( $1440 / \mathrm{mL}$, n.r. 160-400), along with the inversion of the CD4/CD8 ratio $(0.23$, n.r. 1.3-3). Looking for the possible causes of fever of unknown origin, it was initially performed an ultrasound of the abdomen whose outcome was negative. It was thus performed a contrast-enhanced computed tomographic (CT) scan of the abdomen which showed a filling defect of the caudal portion of SMV, with a length of about $5 \mathrm{~cm}$, compatible with a partially occlusive thrombosis (Figure 1).

A screening for thrombophilia was then carried out and revealed the factor $\mathrm{V}$ Leiden heterozygote G1691A mutation, while the test for the prothrombin G20210A mutation was negative, and the levels of anti-cardiolipin and anti- $\beta 2$-glycoprotein antibodies, lupus anticoagulant, antithrombin III, homocysteine, protein $\mathrm{C}$, and $\mathrm{S}$ activity were normal.

In view of the clinical, serological and $\mathrm{CT}$ findings, the diagnosis of acute CMV infection complicated by SMV thrombosis in a patient with factor $\mathrm{V}$ Leiden mutation was made and oral anticoagulant treatment with warfarin was soon started. Two months after the beginning of such therapy, a second contrastenhanced CT scan of the abdomen showed the complete recanalization of SMV (Figure 2). Warfarin therapy was discontinued after 6 months.

\section{Discussion}

This case report is characterized by a diagnosis of SMV thrombosis, which was exclusively found on CT

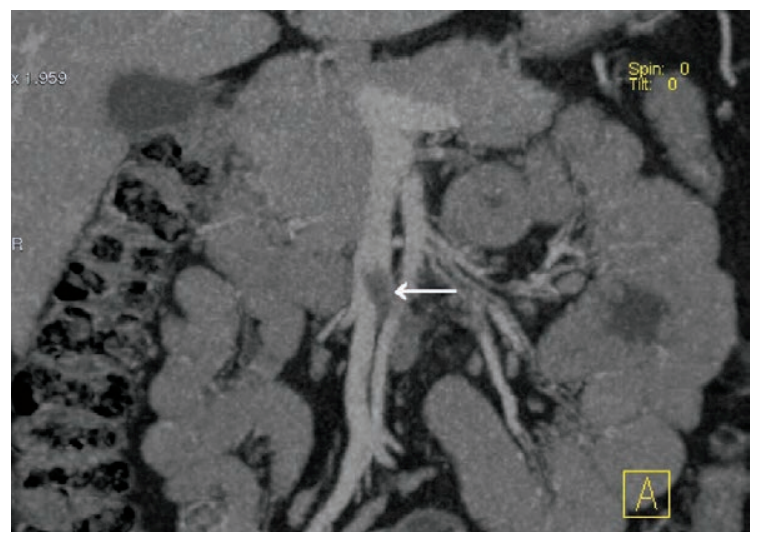

Figure 1. Reconstruction of a computer tomography of the abdomen showing the extent of the thrombus of the superior mesenteric vein (arrow). imaging, in the absence of any abdominal symptom, in an immunocompetent patient with factor $\mathrm{V}$ Leiden mutation who presented with a fever of unknown origin caused by an acute CMV infection. In this regard, it is possible to make three considerations. First, the presence of such inherited pro-thrombotic risk factor (PTRF) favored the appearance of SMV thrombosis in a young man with acute CMV infection and raised the need of an assessment of duration of anticoagulation treatment. Second, the absence of clinical and radiological evidence of portal hypertension was suggestive for both a recent onset and a partial involvement of SMV thrombosis. Third, as the clinical presentation of PS thrombosis is known to depend on the extent of the thrombus, the size of the affected vessel, and the depth of bowel wall ischemia, ${ }^{5}$ in our patient the partial SMV thrombosis could account for both the asymptomatic abdominal clinical picture and its complete recanalization by a prompt treatment with warfarin.

A recent meta-analysis study suggests that the incidence of thrombotic involvement in hospitalized patients with acute CMV infection is $6.4 \%$ and that PS is involved in $37.5 \%$ of immunocompetent patients with acute CMV infection complicated by thrombosis. ${ }^{4}$ The finding of a case of a CMV-associated asymptomatic SMV thrombosis led us to carry out a careful review of the literature for papers concerning thrombotic events of PS in association with acute CMV infection in immunocompetent patients. Interestingly, we retrieved 5 other cases characterized by a partial thrombosis of either portal vein or one of its branches. ${ }^{6-10} \mathrm{In}$ agreement with our case, in 4 of such case reports, patients did not complain of abdominal pain, but presented with a mononucleosis-like syndrome with malaise, prolonged fever, and a variable elevation of aminotransferase levels. ${ }^{7-10}$ With regard to the treat-

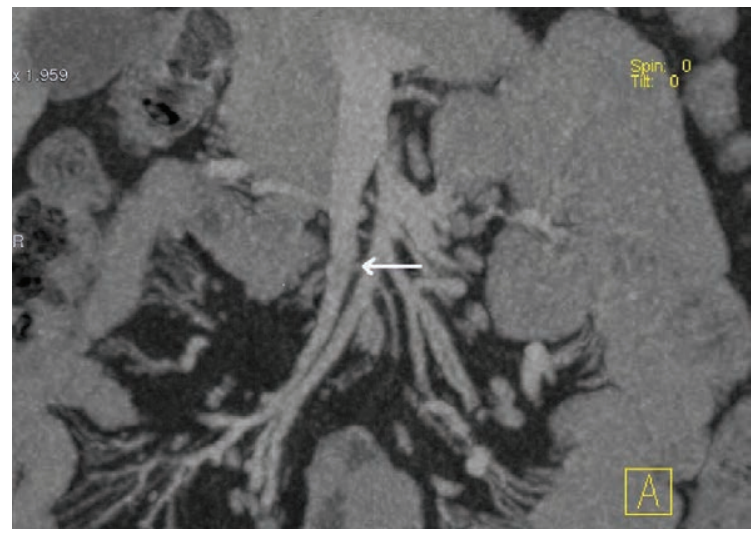

Figure 2. Complete recanalization of the superior mesenteric vein (arrow) two months after oral anticoagulation therapy. 
Table 1. Clinical features of patients with partial thrombosis of portal system associated with acute cytomagalovirus infection.

\begin{tabular}{lcccc}
\hline Pt & Age (years)/Sex & Abdominal symptoms & Thrombosis sites & Treatment and outcome \\
\hline 1 & R $1 / \mathrm{F}$ & R upper quadrant pain; nausea & RB of PV (partial) & Spontaneous resolution at 12 days \\
\hline 2 & $31 / \mathrm{F}$ & None & LB of PV (partial) & Recanalization at 11 months with VKA \\
\hline 3 & $47 / \mathrm{F}$ & None & RB of PV (partial) & Recanalization at 1 week with LMWH relayed with VKA \\
\hline 4 & $29 / \mathrm{M}$ & None & Trunk of PV (partial) & Recanalization at 2 weeks with LMWH relayed with VKA \\
\hline 5 & $34 / \mathrm{M}$ & None & LB of PV (partial) & Recanalization at 10 days with LMWH relayed with VKA \\
\hline 6 & $39 / \mathrm{M}$ & None & SMV (partial) & Recanalization at 2 months with VKA
\end{tabular}

Pt, patient; R, right; RB, right branch; PV, portal vein; LB, left branch; VKA, vitamin K antagonist; LMWH, low molecular weight heparin; SMV, superior mesenteric vein; PC, present case.

ment and the outcome of these cases, a recanalization with low molecular weight heparin and/or vitamin $\mathrm{K}$ antagonists was reported in 5 cases, while a spontaneous resolution was described in one case. Table 1 summarizes their main clinical features. ${ }^{6-10}$

In all the above-mentioned cases portal hypertension was not detected. Therefore, it can be assumed that its absence is to be expected in patients with isolated partial PS thrombosis. We can also infer that in these cases the suspicion index was low and that only diagnostic investigations such as Doppler ultrasonography for PS and contrast-enhanced CT allowed a diagnosis of CMV-associated PS thrombosis. On the whole, these findings are consistent with other studies which suggest that venous thrombosis during acute $\mathrm{CMV}$ infection may be an underestimated complication. ${ }^{2}$

The intrinsic procoagulant effects of CMV infection have been extensively investigated. The non-specific systemic inflammation induced by CMV infection provokes coagulation activation through inflammatory cytokines and proteins synthesis. Moreover, CMV infection of endothelial cells induces expression of adhesion molecules, which enhance platelet and leukocyte adhesion. ${ }^{11}$ There is also evidence that one of the immediate-early gene products of CMV, namely IE84, binds to p53 and inhibits its transcriptional activity, thus inhibiting p53-mediated apoptosis, and enhancing vascular smooth muscle cell proliferation. ${ }^{12}$ Finally, according to the most accepted theory, CMV infection could induce thrombosis through the development of transient anti-phospholipid antibodies (APLA). Interestingly, when the infection resolves APLA usually disappear or their levels decrease. ${ }^{13}$

The link between acute CMV infection and venous thrombosis development still needs to be clarified, although interesting data seem to emerge from a recent study in which PTRFs were investigated. ${ }^{14}$ A remarkable proportion (about two thirds) of immunocompetent patients with such association had either acquired or inherited predispositions for thrombosis. In these patients CMV infection might have acted as the last trigger for thrombosis. On the other hand, about one third of patients with CMV-associated venous thrombosis presented no other PTRFs. Therefore, in these cases CMV infection might have been sufficient by itself to induce thrombosis. On the whole, these data stress the importance of the identification of PTRFs for prevention and treatment strategies. In fact, thrombosis with a transient acquired PTRF, such as CMV infection, may be treated with shorter anticoagulation duration. Conversely, the presence of inherited thrombophilias might require a prolonged anticoagulation treatment. However, in our case Factor V Leiden heterozygosity was not an indication for long-term anticoagulation in accordance with recently published guidelines. ${ }^{15}$

\section{Conclusions}

Even though in immunocompetent patients PS thrombosis can uncommonly complicate an acute CMV infection, physicians should be alert for its diagnostic clues, because an asymptomatic clinical course may occur, especially when there is a partial thrombotic involvement. Moreover, early diagnosis and treatment of PS thrombosis are mandatory because on the one hand the risk of intestinal ischemia and infarction increases with delay of treatment, on the other hand the chance for recanalization is optimized.

\section{References}

1. Wreghitt TG, Teare EI, Sule O, et al. Cytomegalovirus infection in immunocompetent patients. Clin Infect Dis 2003;37:1603-6.

2. Abgueguen P, Delbos V, Ducancelle A, et al. Venous thrombosis in immunocompetent patients with acute cytomegalovirus infection: a complication that may be underestimated. Clin Microbiol Infect 2001;16:851-4.

3. Paran Y, Shalev V, Steinvil A, et al. Thrombosis follow- 
ing acute cytomegalovirus infection: a community prospective study. Ann Hematol 2013;92:969-74.

4. Justo D, Finn T, Atzmony L, et al. Thrombosis associated with acute cytomegalovirus infection: a metaanalysis. Eur J Intern Med 2011;25:195-9.

5. Kumar S, Sarr MG, Kamath PS. Mesenteric venous thrombosis. N Engl J Med 2001;345:1683-8.

6. de Celis G, Mir J, Casal J, Gomez D. 31-year old woman with an enlarged tender liver. Lancet 1995;346:1270.

7. Estival JL, Dehourdeau P, Zammit C, et al. Spontaneous portal vein thrombosis associated with acute cytomegalovirus infection in an immunocompetent patient. Presse Med 2001;30:1876-8.

8. Girszyn N, Leport J, Baux N, et al. Portal vein thrombosis associated with acute cytomegalovirus infection in an immunocompetent patient. Rev Med Interne 2006;27:426-8.

9. Chelbi F, Boutin-Le ThiHuong D, Frigui M, et al. Portal thrombosis complicating an acute cytomegalovirus infection in an immunocompetent patient. Rev Med Interne $2006 ; 27: 54-8$.
10. Squizzato A, Ageno W, Cattaneo A, Brumana N. A case report and literature review of portal vein thrombosis associated with cytomegalovirus infection in immunocompetent patients. Clin Infect Dis 2007:44:e13-6.

11. Tichelaar VY, Kluin-Nelemans HJ, Meijer K. Infections and inflammatory diseases as risk factors for venous thrombosis. A systematic review. Thromb Haemost 2012; 107:827:37.

12. Speir E, Modali R, Huang ES, et al. Potential role of human cytomegalovirus and $\mathrm{p} 53$ interaction in coronary restenosis. Science 1994;265:391-4.

13. Labarca JA, Rabaggliati RM, Radrigan FJ, et al. Antiphospholipid syndrome associated with cytomegalovirus infection: case report and review. Clin Infect Dis 1997;24:197-200.

14. Atzmony L, Grosfeld A, Saar N, Justo D. Inherited and acquired predispositions for thrombosis in immunocompetent patients with cytomegalovirus-associated infection. Eur J Intern Med 2010;21:2-5.

15. Kujovich JL. Factor V Leiden thrombophilia. Genet Med 2011;13:1-16. 